\section{Demandas laborales y consumo de alcohol: el rol del grupo de trabajo}

\author{
Work demands and alcohol consumption: \\ the job group's role
}

\section{Exigências laborais e consumo de álcool: o papel do grupo de trabalho}

\author{
Marjory Güilgüiruca 1 \\ Marcela Quiñones 1 \\ Claudia Zúñiga 1
}

\section{Resumen}

El objetivo del presente estudio fue determinar si la identificación con grupos de trabajo, con normas desfavorables y favorables de consumo de alcohol del grupo de trabajo, modera la relación entre las demandas del trabajo y el consumo de alcohol en trabajadores. A través de una análisis de regresión, usando la macro de moderación moderada en una muestra de trabajadores de una empresa de telecomunicaciones de Chile $(N=1.143)$, se presta apoyo a las hipótesis de moderación moderada: la alta identificación con grupos de trabajo con normas desfavorables de consumo de alcohol de ese grupo amortigua la relación entre las demandas del trabajo y el consumo de alcohol en trabajadores ( $B=-0,080 ; t=-2,01 ; p=0,04$; IC95\%: -0,1600; -0,0024) y la alta identificación con grupos de trabajo con normas favorables de consumo de alcohol de ese grupo incrementa la relación entre las demandas del trabajo y el consumo de alcohol en trabajadores ( $B=0,170 ; t=2,36 ; p=0,01 ;$ IC95\%: 0,030; 0,322). Estos resultados sugieren que la alta identificación con grupos de trabajo y sus normas sociales de consumo de alcohol son recursos laborales que, de manera conjunta, pueden afectar o proteger a trabajadores expuestos a altas demandas laborales. Por lo tanto, se recomienda atender a los potenciales efectos positivos, así como también a los efectos perniciosos, de estos recursos laborales.

Estrés Laboral; Consumo de Bebidas Alcohólicas; Identificación Social; Normas Sociales

\author{
Correspondencia \\ M. Güilgüiruca \\ Universidad de Chile. \\ Av. C. Ignacio Carrera Pinto 1045, Santiago 6850331, Chile. \\ mguilgui@ug.uchile.cl \\ 1 Universidad de Chile, Santiago, Chile.
}




\section{Introducción}

El consumo de alcohol es considerado uno de los principales factores de riesgo para la salud pública 1. En efecto, es responsable de 33 millones de muertes anuales en el mundo ${ }^{1}$, es factor causal en más de 200 enfermedades y trastornos y es reconocido por incidir en la discapacidad y muerte de personas en edad productiva 2

Asimismo, el consumo de alcohol es un importante problema para las organizaciones, por sus efectos en el ausentismo 3,4 , los accidentes del trabajo 5 , la productividad y las pérdidas económicas 6 , entre otras consecuencias.

La relación entre los factores organizacionales y el consumo de alcohol en trabajadores ha sido bastante atendida por la literatura, no obstante, la evidencia aún es inconsistente 7. Por ejemplo, existen numerosos estudios que plantean que elevadas demandas laborales y bajo control en el trabajo 8,9,10, altos niveles de esfuerzo, combinados con bajas retribuciones 11,12,13,14, así como bajas exigencias laborales y baja capacidad para tomar decisiones $15,16,17$, se relacionan con el consumo de alcohol en trabajadores, no obstante, también hay hallazgos que sostienen que esta asociación es casi inexistente 18,19,20.

Considerando la naturaleza mixta de la evidencia, la presente investigación plantea que una mayor comprensión del problema podría contemplar aspectos menos atendidos por la literatura como son: el sentido de pertenencia del grupo de trabajo y sus normas de consumo. De este modo, basándonos en el modelo Demandas-Recursos Laborales (DRL) 21,22 y la Teoría de la Identidad Social (TIS) 23,24, planteamos que el consumo de alcohol podría estar explicado por la exposición de los trabajadores a altas demandas laborales y a la combinación entre una elevada identificación con el grupo de trabajo y las normas de consumo de alcohol de este grupo.

Cabe señalar que hasta la fecha no se conocen investigaciones que analicen el efecto conjunto de estos tres predictores en el consumo de alcohol de trabajadores, no obstante, la literatura ofrece diversa evidencia que apunta en esta dirección. Por ejemplo, se ha descubierto que el binomio altas demandas laborales y alta identificación con el trabajo 25, así como altas demandas laborales y normas de alto consumo de alcohol de los compañeros 26 , incrementó el consumo de alcohol de los trabajadores.

Asimismo, en contextos universitarios se descubrió que la alta identificación con líderes estudiantiles con normas de bajo consumo de alcohol amortiguó el consumo de esta sustancia en estudiantes 27,28,29, y que una elevada identificación con estudiantes con normas de alto consumo de alcohol acentuó su ingesta 30,31,32. De este modo, los hallazgos descritos nos permiten inferir que podría encontrarse un efecto similar en el contexto laboral.

De acuerdo al modelo DRL 21,22, el bienestar del trabajador depende de dos aspectos: las demandas y los recursos laborales. Las demandas laborales son aspectos psicológicos, físicos, sociales u organizacionales del trabajo que implican costos emocionales, cognitivos o físicos para el trabajador 21,22, como el burnout, la depresión 33,34, la ansiedad en el trabajo, la fatiga mental y la insatisfacción laboral 35 .

Por otra parte, los recursos laborales son aspectos psicológicos, físicos, sociales u organizacionales que gatillan procesos motivacionales, así como protegen de las consecuencias negativas de las demandas 22. En efecto, las demandas y los recursos laborales, al interactuar entre sí, atenúan el impacto negativo de las demandas del trabajo, así como amplifican el efecto de estas en el bienestar del trabajador 22 .

Un factor que podría actuar como un recurso del trabajador es la identidad social. Según la TIS 23,24 , la identidad social es la parte del auto-concepto derivada de la conciencia de pertenencia a una o más categorías sociales 23,24 . Este proceso reviste complejidad, porque solo aquellos grupos, que tienen una mayor significación psicológica para el individuo, podrán ejercer influencia en su conducta por intermedio de las normas sociales 36,37,38 en este caso de consumo de alcohol o no.

En este sentido, existen estudios que muestran que elevados niveles de identificación grupal se relacionan con una mayor aceptación y cumplimiento de las normas del grupo 39,40 , entendidas como las reglas de comportamientos que son socialmente aceptadas por el propio grupo 41 .

El presente estudio aborda la norma descriptiva, entendida como lo que la mayoría de las personas de un grupo hace 42 . Esta norma ha acumulado importante evidencia por su impacto en el comportamiento humano 43,44 , y su principal atributo es que es mucho menos susceptible a las reper- 
cusiones sociales que la norma prescriptiva 45 , que alude a reglas de conducta moralmente aprobadas y/o reprobadas por el grupo 42.

En el ámbito laboral, la identificación con el grupo de trabajo es una forma específica de identidad social que supone la pertenencia a un grupo de trabajo particular 46,47 . La importancia de este factor se debe a diversos motivos, por ejemplo, a que los procesos de identificación social son más fuertes en grupos más pequeños que en grupos más amplios 48, se ha asociado a indicadores positivos de bienestar 49,50,51,52 y ha demostrado ser un efectivo reductor de las demandas laborales 53 . No obstante lo anterior, la literatura advierte que la pertenencia a ciertos grupos también puede asociarse a indicadores perjudiciales de salud, a través del capital social negativo 54 , lo que podría deberse a la internalización voluntaria de normas de salud contraproducentes 55 .

Considerando los antecedentes descritos planteamos las siguientes hipótesis: (H1) la alta identificación con un grupo de trabajo con normas de bajo consumo de alcohol amortiguará la relación entre las demandas laborales y el consumo de alcohol de los trabajadores; (H2) la alta identificación con un grupo de trabajo con normas de alto consumo de alcohol amplificará la relación entre las demandas laborales y el consumo de alcohol de los trabajadores; (H3) la baja identificación con un grupo de trabajo no intervendrá en la relación entre las demandas laborales y el consumo de alcohol de los trabajadores, independiente de sus normas hacia el consumo de alcohol.

El modelo teórico se presenta en la Figura 1.

\section{Método}

\section{Diseño y procedimiento}

El estudio se realizó en una empresa chilena de telecomunicaciones con presencia nacional. La ejecución del estudio se realizó en coordinación con la Unidad de Calidad de Vida de la empresa.

El diseño fue transversal, no experimental, selectivo de encuesta 56. La recolección de los datos se realizó a través de una encuesta online. Se extendió una invitación general a los trabajadores y quienes

Figura 1

Modelo conceptual del efecto de moderación moderada de la identificación con el grupo de trabajo y la norma de consumo de alcohol, en la relación entre las demandas laborales y el consumo de alcohol de los trabajadores.

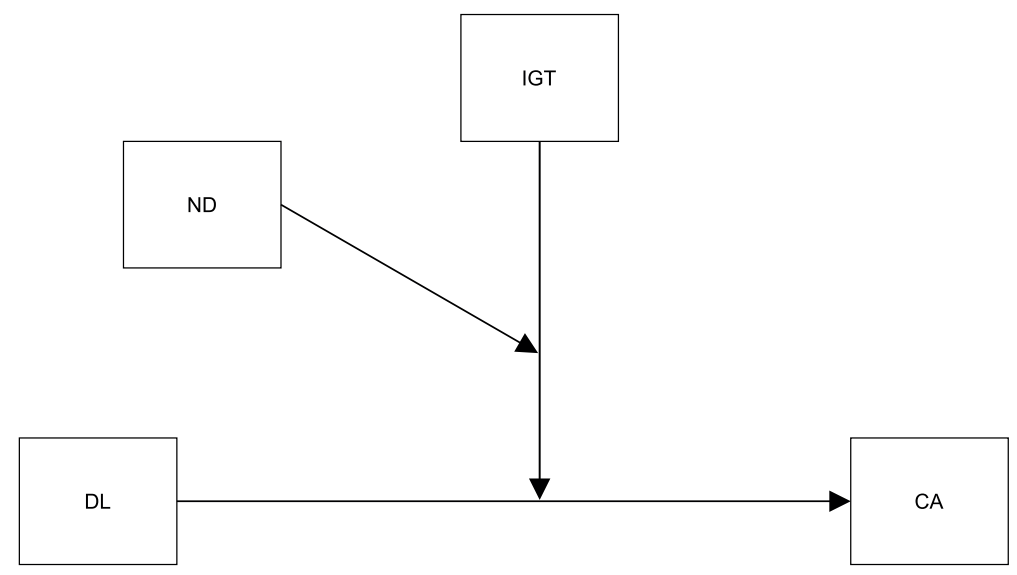

CA: consumo de alcohol del trabajador; DL: demanda laboral; IGT: identidad con el grupo de trabajo; ND: norma descriptiva de consumo de alcohol del grupo de trabajo. 
aceptaron participar firmaron un consentimiento informado, el cual explicaba el objetivo del estudio, los procedimientos y el manejo confidencial de los datos. La devolución de resultados fue dirigida a la Unidad de Calidad de Vida y a los propios participantes que señalaron su interés en conocerlos.

\section{Participantes}

Se invitó a participar a 4.048 trabajadores, de los cuales 1.361 respondieron el cuestionario, lo que implica una tasa de respuesta de 33,6\%. De los cuestionarios contestados 218 no fueron considerados, por poseer respuestas incompletas en alguno de los instrumentos, generando una muestra final de 1.143 personas. Un 64,6\% de los participantes eran hombres (734) y 35,8\% eran mujeres (409). La edad promedio fue de 43 años (DT = 9.0) y fluctuaron entre 18 años y 65 años. El 69,7\% culminó estudios universitarios y/o técnicos, el 11,4\% desarrolló estudios de postgrado y el 18,9\% presentó estudios técnicos y universitarios incompletos. El promedio de antigüedad laboral fue de 11,8 años (DT $=7,0)$ y la jornada de trabajo predominante fue la diurna (60,6\%).

Para determinar el nivel de consumo de alcohol de la muestra, se utilizó la clasificación para población chilena del Alcohol Use Disorders Identification Test (AUDIT) 57. En base a estos criterios, se encontró que la mayoría de los participantes no reportaron problemas con el consumo, presentando solo un 5,9\% consumo riesgoso, y un 3,6\% consumo perjudicial o dependencia. Entre los hombres el 4,4\% presentó consumo riesgoso y el 2,9\% presentó consumo perjudicial o dependencia y entre las mujeres el 1,6\% presentó consumo riesgoso y el 0,7\% presentó consumo perjudicial o dependencia.

En cuanto al nivel de educación según tipo de consumo, es posible señalar que de los trabajadores con consumo riesgoso, el 0,8\% tiene educación técnica y universitaria incompleta, el 4,1\% posee educación técnica o universitaria completa y el 1\% posee estudios de posgrado. Y de los trabajadores con consumo perjudicial y dependencia el 0,4\% tiene educación técnica y universitaria incompleta, 2.4\% posee educación técnica y universitaria completa y $0,7 \%$ posgrado.

\section{Instrumentos}

\section{- Demandas laborales extrínsecas}

Se utilizó la escala de esfuerzo extrínseco del cuestionario Desequilibrio Esfuerzo-Recompensa de Siegrist et al. 58. Esta escala evalúa en seis ítems las demandas u obligaciones que son impuestas a los trabajadores, en forma de número de tareas, ritmo de trabajo e interrupciones. Para muestras de trabajadores de oficina se recomienda considerar solo cinco ítems, suprimiendo la afirmación "Mi trabajo es físicamente exigente" por ser poco pertinente. La escala fue traducida al español por las autoras ${ }^{59}$, quienes poseen experiencia en traducción y en adaptación de instrumentos psicométricos. Ejemplos de ítems son: "Normalmente tengo que trabajar contra el tiempo" y "En el último tiempo mi trabajo se ha vuelto cada vez más exigente". Las alternativas de respuesta fueron muy en desacuerdo, en desacuerdo, de acuerdo y muy de acuerdo.

\section{- Demandas laborales emocionales}

Se utilizaron cuatro preguntas del Cuestionario SUSESO-ISTAS 21 - versión chilena, validada a población chilena por Alvarado et al. 60. Las preguntas evalúan carga emocional, entendida como agobio o desgaste, y exigencia de ocultar opiniones y emociones en el trabajo. En la escala original los ítems se plantean en forma de preguntas, por lo que fueron transformadas en afirmaciones por las autoras 59 para homologar el formato con el resto de los instrumentos aplicados. Ejemplos de ítems son: "En general mi trabajo me produce desgaste emocional" y "A veces tengo que guardarme mis opiniones en el trabajo". Las alternativas de respuesta fueron muy en desacuerdo, en desacuerdo, de acuerdo y muy de acuerdo. 


\section{- Consumo de alcohol}

Se utilizó el AUDIT, en su versión validada en Chile por Alvarado et al. 57. Esta medida evalúa el consumo problemático de alcohol por medio de diez preguntas. Ejemplos de estas preguntas son: “¿Qué tan seguido toma usted alguna bebida alcohólica?” “Qué tan seguido, en los últimos 12 meses, usted no pudo parar de beber una vez que había empezado?”. Las alternativas de respuesta siguen una lógica de escala Likert ( 0 a 4 puntos), que evalúan cantidad y frecuencia de eventos relacionados con el consumo de alcohol.

\section{- Identificación con el grupo de trabajo}

Se utilizó la medida de ítem único propuesta por Postmes et al. 61. El ítem original "I identify with [ingroup]" fue traducido y adaptado por las autoras 59 como "Me identifico con los miembros de mi grupo de trabajo" y las alternativas de respuesta fueron cinco (totalmente en desacuerdo, desacuerdo, ni de acuerdo ni en desacuerdo, de acuerdo y totalmente de acuerdo).

\section{- Norma descriptiva}

Se utilizó la escala de Rimal \& Real 62, que mide la percepción individual de la cantidad de consumo de alcohol del grupo. La escala posee cuatro ítems que fueron traducidos y adaptados por las autoras quienes poseen experiencia en traducción y adaptación de instrumentos psicométricos 59. Ejemplos de los ítems son: "Cuando un típico miembro de su grupo de trabajo va a un bar ¿cuántos tragos cree que consume?” " “Cuando un típico miembro de su grupo de trabajo va a una fiesta ¿cuántos tragos cree que consume?". Las preguntas tuvieron seis alternativas de respuestas que van desde "0" hasta "9 y más tragos”.

\section{- Variables de control}

Se decidió controlar las siguientes variables: sexo (mujer; hombre); edad (42 años y menos; 43 años y más) y depresión, (con y sin diagnóstico de depresión el último año) por ser predictoras reconocidas del consumo de alcohol 63,64.

\section{Estrategia de análisis}

\section{- Análisis factorial confirmatorio}

Con el objetivo de revisar la validez de las medidas utilizadas se realizó un análisis factorial confirmatorio (AFC), utilizando el software estadístico Mplus versión 7.0 (https://www.statmodel.com/). Se utilizó el Método Mínimos Cuadrados no Ponderados Robusto (ULSMV), estimador recomendado para variables ordinales, distribuciones no normales y basado en la matriz de correlaciones policóricas 65 . Se evaluó el modelo considerando cuatro factores (demandas laborales; identificación con el grupo de trabajo, norma descriptiva del consumo de alcohol del grupo de trabajo y consumo de alcohol del trabajador). Para evaluar el ajuste del modelo se consideraron valores de $\chi^{2}$ no significativo, índice de ajuste absoluto RMSEA entre < 0,05 y 0,08 e índices de ajuste incremental CFI, TLI, $>0,9565$. Posteriormente, se evaluó la consistencia interna de las escalas, por medio del coeficiente alpha ordinal para variables categóricas, siendo 0,70 un valor de alpha óptimo 66.

\section{- Análisis de moderación moderada}

Este procedimiento examina el efecto de dos moderadores $\mathrm{Z}$ y W en la relación entre una variable independiente $\mathrm{X}$ y una variable dependiente $\mathrm{Y}$ 67. Cuando los dos moderadores (identificación con el grupo de trabajo y norma de consumo de alcohol de este grupo) debilitan la relación entre X e Y se denomina efecto de amortiguación y cuando ambos moderadores intensifican esta relación se denomina efecto de amplificación. 
Para testear esta interacción se utilizó la macro PROCESS (http://www.processmacro.org/) para SPSS versión 18 (https://www.ibm.com/). Se seleccionó la técnica pick a point que divide las variables moderadoras en dos categorías (eg. alta y baja identificación con el grupo de trabajo; y altas y bajas normas de consumo de alcohol del grupo de trabajo (equivalente a norma favorable y desfavorable hacia el consumo de alcohol). El análisis de los procesos condicionales permite detectar en qué niveles de las variables moderadoras ocurren las interacciones planteadas. Para corroborar la existencia de moderación moderada, se utilizarán dos criterios: el valor de p de la interacción entre las demandas laborales, la identificación con el grupo de trabajo y la norma del grupo sobre el consumo de alcohol y los intervalos de confianza de las interacciones según la técnica de bootstraping.

\section{Resultados}

De acuerdo con los resultados del análisis factorial confirmatorio, el modelo propuesto de cuatro factores obtuvo un ajuste adecuado $\left(\chi^{2}=458,068(225)\right.$; $\mathrm{RMSEA}=0,030$; $\mathrm{CFI}=0,95$; TLI $\left.=0,94\right)$. Del mismo modo, todos los ítems presentaron adecuadas cargas factoriales en sus respectivos factores $\geq$ $0,55(\mathrm{p}=0,00)$. Como puede observarse en la Tabla 1, las escalas presentaron confiabilidades elevadas, con valores alpha ordinales superiores a 0,90. Estos resultados corroboran las adecuadas propiedades psicométricas de las escalas utilizadas, así como la idoneidad de la estructura teórica planteada.

Por otra parte, la matriz de correlaciones policóricas (Tabla 1) muestra que todas las variables se relacionan de forma significativa. Las demandas laborales y las normas de consumo del grupo de trabajo se asocian de forma positiva y significativa con el consumo de alcohol de los empleados, mientras que la identificación con el grupo de trabajo se relaciona negativa y significativamente con el consumo de alcohol de los trabajadores.

\section{Análisis de moderación moderada}

El modelo testeado muestra que la asociación entre las demandas laborales (extrínsecas y emocionales) y el consumo de alcohol de los trabajadores, está moderada por la identificación con el grupo de trabajo (alta y baja identificación) y las normas de consumo de alcohol por parte del grupo $\mathrm{F}$ $(13,1129)=13,26 ; \mathrm{p}<0,01 ; \mathrm{R}^{2}=0,27$.

La parte intermedia de la Tabla 2, muestra dos efectos de interacción que explican significativamente el consumo de alcohol de los trabajadores. El primero corresponde al efecto de moderación moderada, representado por la interacción significativa entre las demandas laborales, la identificación con el grupo de trabajo y la norma descriptiva de consumo de alcohol del grupo de trabajo, como variables predictoras del consumo de alcohol $(B=0,03 ; t=2,15 ; p=0,03 ;$ IC95\%: 0,002; 0,049). El segundo, muestra el efecto de moderación simple entre la identificación con el grupo de trabajo y la norma descriptiva de consumo de alcohol como predictoras del consumo de alcohol de los trabajadores $(B=0,09 ; t=2,12 ; p=0,03 ;$ IC95\%: 0,007; 0,183).

\section{Tabla 1}

Estadísticos descriptivos, matriz de correlaciones y coeficientes de confiabilidad ( $\mathrm{N}=1.143)$.

\begin{tabular}{|c|c|c|c|c|c|c|c|}
\hline & & Media & DT & 1 & 2 & 3 & 4 \\
\hline 1 & DL & 2,63 & 0,54 & 0,92 & & & \\
\hline 2 & IGT * & 4,02 & 0,85 & $-0,45 * *$ & & & \\
\hline 3 & ND & 1,40 & 0,69 & 0,24 ** & $-0,16$ ** & 0,95 & \\
\hline 4 & CA & 0,24 & 0,26 & $0,23 * *$ & $-0,17 * *$ & $0,51 * *$ & 0,93 \\
\hline
\end{tabular}

CA: consumo de alcohol del trabajador; DL: demanda laboral; DT: desviación típica; IGT: identidad con el grupo de trabajo; ND: norma descriptiva de consumo de alcohol del grupo de trabajo.

* IGT sin cálculo de confiabilidad por ser medida de ítem único.

** La correlación es significativa a nivel 0,05 (bilateral). 


\section{Tabla 2}

Efectos simples y de interacción de las demandas laborales en el consumo de alcohol, considerando la identificación con el grupo de trabajo y la norma de consumo $(\mathrm{N}=1.143)$.

\begin{tabular}{|c|c|c|c|c|c|}
\hline & B & SE & $\mathbf{t}$ & Valor de p & $\begin{array}{c}\text { Bootstrap } \\
\text { (IC95\%) }\end{array}$ \\
\hline \multicolumn{6}{|l|}{ Efectos simples } \\
\hline $\mathrm{DL}$ & 0,0100 & 0,02 & 0,88 & 0,37 & $-0,020 ; 0,060$ \\
\hline IGT & $-0,2500$ & 0,10 & $-2,52$ & 0,01 * & $-0,459 ;-0,057$ \\
\hline ND & 0,3500 & 0,04 & 8,66 & 0,00 * & 0,$270 ; 0,429$ \\
\hline \multicolumn{6}{|c|}{ Efectos de interacción } \\
\hline DL $\times$ IGT $\times$ ND & 0,0256 & 0,01 & 2,15 & $0,03 * \star$ & 0,$002 ; 0,049$ \\
\hline IGT x ND & 0,0950 & 0,04 & 2,12 & $0,03 * *$ & 0,$007 ; 0,183$ \\
\hline $\mathrm{DL} \times \mathrm{IGT}$ & 0,0319 & 0,02 & 1,47 & 0,14 & $-0,011 ; 0,074$ \\
\hline $\mathrm{DL} \times \mathrm{ND}$ & 0,0252 & 0,01 & 1,95 & 0,05 & 0,$000 ; 0,050$ \\
\hline \multicolumn{6}{|l|}{ Covariables } \\
\hline Sexo & 1,0900 & 0,14 & 7,60 & $0,00 * *$ & 0,$811 ; 1,380$ \\
\hline Depresión & 1,0400 & 0,45 & 2,30 & $0,02 * \star$ & 0,$152 ; 1,937$ \\
\hline Edad & $-0,4900$ & 0,17 & $-2,87$ & $0,00 * *$ & $-0,828 ;-0,156$ \\
\hline $\mathrm{R}^{2}$ & 0,2700 & & & & \\
\hline $\mathrm{F}$ & 13,2600 & & & & \\
\hline
\end{tabular}

B: coeficiente de regresión no estandarizado; DL: demanda laboral; F: estadístico F; IC95\%: intervalo del 95\% de confianza; IGT: identidad con el grupo de trabajo; ND: norma descriptiva de consumo de alcohol del grupo de trabajo; SE: error estándar; t: estadístico t.

$* \mathrm{p}<0,01$;

** $\mathrm{p}<0,05$.

En la Tabla 3 se muestran los efectos condicionales que ejercen las demandas laborales para explicar el consumo de alcohol de los trabajadores, en base a los niveles de la identificación con el grupo de trabajo y los niveles de las normas de consumo de alcohol del grupo de trabajo. En primer lugar, se observa que la relación entre las demandas laborales y el consumo de alcohol es amortiguada cuando existe una alta identificación con el grupo de trabajo y este grupo presenta una norma desfavorable del consumo de alcohol ( $\mathrm{B}=-0,080 ; \mathrm{t}=-2,01 ; \mathrm{p}=0,04$; IC95\%: -0,1600; -0,0024), aportando evidencia a favor de la hipótesis 1 . En segundo lugar, la relación entre las demandas laborales y el consumo de alcohol se ve amplificada cuando existe una alta identificación con el grupo de trabajo y este presenta normas favorables al consumo de alcohol ( $\mathrm{B}=0,170 ; \mathrm{t}=2,36 ; \mathrm{p}=0,01$; IC95\%: 0,0300; 0.322), dando apoyo a la hipótesis 2 .

Finalmente, y de acuerdo con lo propuesto en la hipótesis 3, la baja identificación con el grupo de trabajo no desempeñó un efecto moderador en la relación entre demandas laborales y consumo de alcohol, en ninguna de las dos condiciones: norma de consumo desfavorable $(\mathrm{B}=-0,017 ; \mathrm{t}=$ $-0,51 ; \mathrm{p}=0,60)$ y favorable $(\mathrm{B}=0,000 ; \mathrm{t}=-0,00 ; \mathrm{p}=0,99)$.

La Figura 2 muestra la representación gráfica de las interacciones planteadas por el estudio. En la parte superior se observa el efecto de amplificación, representado por la alta identificación con el grupo de trabajo y la norma favorable de consumo de alcohol, que incrementan la relación entre las demandas laborales y el consumo de alcohol del trabajador. En la parte inferior de la figura, se observa el efecto de amortiguación, que muestra que la alta identificación con el grupo de trabajo y sus normas desfavorables de consumo de alcohol disminuyen la relación entre las demandas laborales y el consumo de alcohol del trabajador. 
Tabla 3

Efectos condicionales de las demandas laborales en el consumo de alcohol para valores específicos de identidad con el grupo de trabajo en condiciones de normas favorables y desfavorables de consumo de alcohol del grupo de trabajo.

\begin{tabular}{lcccccc}
\hline IGT & ND & Coeficiente & SE & t & Valor de p & Bootstrap (IC95\%) \\
\hline Alta & Desfavorable & $-0,080$ & 0,04 & $-2,01$ & $0,04 *$ & $-0,167 ;-0,002$ \\
Alta & Favorable & 0,170 & 0,07 & 2,36 & $0,01 *$ & 0,$030 ; 0,322$ \\
Baja & Desfavorable & $-0,017$ & 0,03 & $-0,51$ & 0,60 & $-0,087 ; 0,050$ \\
Baja & Favorable & 0,000 & 0,05 & $-0,00$ & 0,99 & $-0,100 ; 0,104$ \\
\hline
\end{tabular}

IC95\%: intervalo del 95\% de confianza; IGT: identidad con el grupo de trabajo; ND: norma descriptiva de consumo de alcohol del grupo de trabajo; SE: error estándar; t: estadístico t.

$\star p<0,05$.

\section{Figura 2}

Efecto de moderación moderada de la identificación con el grupo de trabajo y la norma de consumo de alcohol del grupo de trabajo en la relación entre demandas laborales y consumo de alcohol del trabajador.
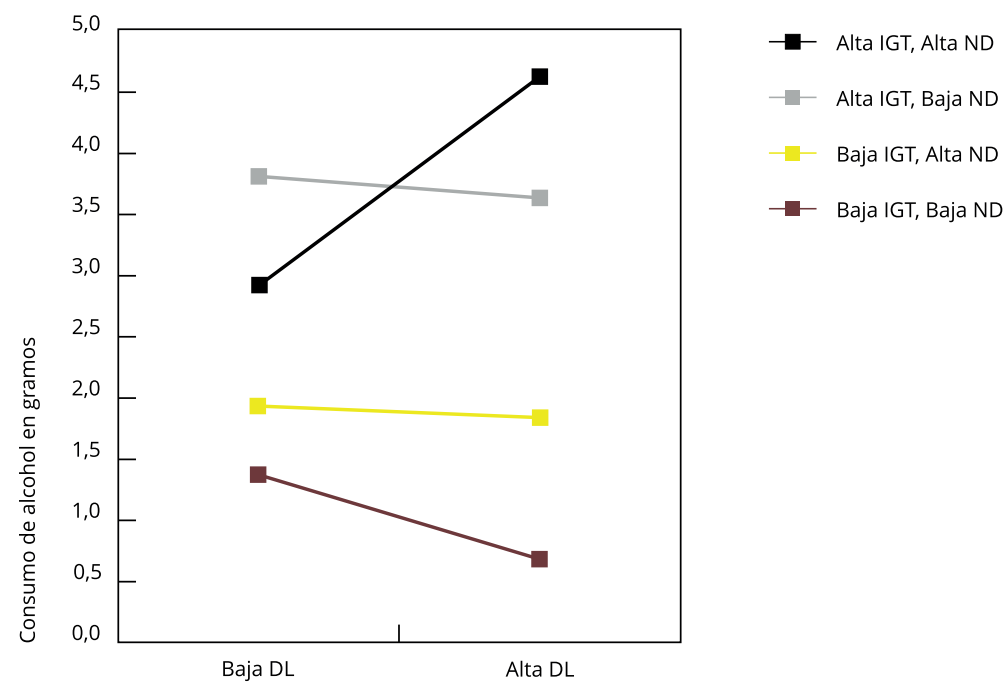

DL: demanda laboral; IGT: identidad con el grupo de trabajo; ND: norma descriptiva de consumo de alcohol del grupo de trabajo.

\section{Discusión}

Este estudio tuvo como objetivo determinar si la identificación con el grupo de trabajo, en conjunto con las normas de consumo de alcohol del mismo grupo, moderan la relación entre las demandas laborales y el consumo de alcohol de los trabajadores.

Los resultados de esta investigación permiten confirmar este objetivo, a través de las tres hipótesis propuestas. En primer lugar, se confirmó la hipótesis de amortiguación, es decir, aquellos empleados que se identificaron fuertemente con grupos de trabajo con normas sociales contrarias al consumo de alcohol, presentaron una menor ingesta de alcohol, como efecto de las demandas laborales. 
En segundo lugar, se confirmó la hipótesis de amplificación, esto es, trabajadores altamente identificados con grupos de trabajo con normas favorables al consumo de alcohol, presentaron un mayor consumo de esta sustancia como efecto de las demandas laborales.

Desde la tradición de la identidad social estos resultados se explicarían por la internalización de la norma social $23,24,37,38$, ya que cuando el individuo se autocategoriza como miembro de un grupo es muy probable que internalice como propios los valores y creencias del grupo y oriente su comportamiento en función de esas creencias.

El efecto de amortiguación observado indica que la alta identificación con grupos de trabajo que poseen normas más saludables puede ser un recurso que actúa como factor protector para la salud, haciendo a los individuos menos susceptibles a los efectos negativos del trabajo. Estos resultados están en línea con los planteamientos del modelo DRL, donde los recursos laborales actúan como reductores de los efectos nocivos de las altas demandas del trabajo 21,22. En efecto, la identificación con este tipo de grupo de trabajo redundaría en beneficios, tanto personales como organizacionales, ya que por un lado contribuye a la salud del trabajador y por otro, permite sostener las demandas del trabajo sin afectar los objetivos organizacionales.

El efecto amplificador encontrado sugiere que la identificación con grupos de trabajo que valoran positivamente el consumo de alcohol sería un factor que hace al trabajador más vulnerable al impacto negativo de las demandas laborales, promoviendo un mayor consumo de alcohol. Estos hallazgos están en línea con el concepto de capital social negativo, donde la sola pertenencia a ciertos grupos conlleva peores indicadores de salud 54 , lo que según la TIS se debe a la internalización voluntaria de normas perjudiciales que conducen al individuo a comportamientos negativos de salud, por su búsqueda de pertenencia social 55 .

Cabe señalar que la hipótesis de amplificación representa un punto de debate para la noción de recursos del enfoque DRL. Desde esta perspectiva, los recursos son concebidos como factores protectores, que conllevan sólo impactos positivos para la salud 22. Por ello, la confirmación de la hipótesis de amplificación con resultado negativo para la salud advierte, a nuestro entender por primera vez, de la existencia de recursos "condicionales". Es decir, factores sociales que podrían reducir los efectos de las demandas laborales, bajo determinadas circunstancias, podrían adoptar un papel contraproducente para la salud.

También se encontró evidencia favorable a la tercera hipótesis, es decir, la baja identificación con el grupo de trabajo no intervino en la relación entre las demandas laborales y el consumo de alcohol de los participantes, independiente de las normas sociales de consumo de alcohol del grupo. Este hallazgo es coherente con lo propuesto por la TIS, en relación a que sólo aquellos grupos psicológicamente relevantes para el individuo tendrán un impacto en su conducta 36,37,38. De este modo, grupos sin relevancia para el individuo no incidirán en su comportamiento de consumo.

Este estudio presenta contribuciones teóricas y prácticas importantes. Dentro de las primeras, se destaca la inclusión de variables psicosociales en la relación demandas laborales-consumo de alcohol. Esto recoge planteamientos iniciales de Frone et al. ${ }^{25}$, en cuanto a que factores psicosociales podrían contribuir a explicar esta relación, motivando una visión más integral del consumo de alcohol en población trabajadora.

A su vez, este estudio advierte de la existencia de recursos laborales con una naturaleza condicionada, es decir, bajo ciertas circunstancias, algunos factores pueden tanto favorecer la salud como perjudicarla. Por lo tanto, se sugiere reconsiderar la propiedad exclusivamente beneficiosa de los recursos propuesta por el enfoque DRL y atender también a sus efectos perniciosos.

Por otra parte, en términos aplicados, este estudio aporta al desarrollo de un enfoque de intervención y prevención más integral del consumo de alcohol en el trabajo. El reconocer grupos de trabajo menos saludables podría ser una oportunidad para intervenir y modificar las normas del grupo, conservando o fortaleciendo los procesos de identificación social de los trabajadores, lo que beneficiaría a la organización y a sus miembros. En esta línea se encuentran algunos estudios experimentales que por medio de intervenciones basadas en las normas de consumo, disminuyeron con éxito conductas de consumo de alcohol en adolescentes y adultos 68,69 .

En cuanto a las limitaciones del estudio, cabe recordar que el diseño transversal utilizado imposibilita plantear inferencias causales entre las variables estudiadas, por lo que se sugiere considerar en el futuro diseños longitudinales o experimentales, que puedan avanzar hacia una comprensión causal 
del fenómeno. En segundo lugar, debido a que la muestra presentó mayoritariamente bajos niveles de consumo de alcohol, se sugiere replicar este estudio en poblaciones con mayor prevalencia del consumo de alcohol, así como seguir los planteamientos de Reis et al. 70 respecto a estratificar los resultados por grupos de consumo de alcohol. A pesar de las limitaciones planteadas, los efectos de interacción testeados fueron significativos por lo que somos optimistas respecto a futuros hallazgos.

Por otro lado, para disminuir los posibles sesgos que presentan las medidas de autoreporte del consumo de alcohol, se recomienda introducir el uso de indicadores psicofisiológicos como medidas complementarias. Se sugiere también explorar y comparar diversas formas de identificación social al interior de la organización, como por ejemplo grupos profesionales o de igual ocupación, con el objetivo de diferenciar entre colectivos con mayor o menor potencial para influir en el comportamiento de consumo de los individuos.

Finalmente, se puede concluir que la identidad social constituye una perspectiva fértil para el estudio de indicadores de salud en trabajadores expuestos a condiciones laborales adversas, representando una comprensión integral de los efectos del trabajo en la salud, que puede ser provechosa para futuras investigaciones e intervenciones organizacionales.

\section{Colaboradores}

M. Güilgüiruca, Marcela Quiñones y Claudia Zúñiga contribuyeron sustancialmente a la obra, en el análisis e interpretación de los datos, en la redacción del trabajo y revisión crítica del contenido intelectual y en la aprobación de la versión final de la obra.

\section{Informaciones adicionales}

ORCID: Marjory Güilgüiruca (0000-0001-8154682X); Marcela Quiñones (0000-0001-7598-4751); Claudia Zúñiga (0000-0003-1691-5742).

\section{Agradecimientos}

Marjory Güilgüiruca agradece a la beca CONICYT no 21120642 (Comisión Nacional de (Comisión Nacional de Investigación Científica y Tecnológica) para estudios de Doctorados Nacionales.

\section{Referencias}

1. World Health Organization. Global status report on alcohol and health 2014. Geneva: World Health Organization; 2014.

2. Organización Panamericana de la Salud. Informe de situación regional sobre el alcohol y la salud en las Américas. Enfermedades no transmisibles y salud mental, Washington DC: Organización Panamericana de la Salud; 2015.

3. Bacharach SB, Bamberger P, Biron M. Alcohol consumption and workplace absenteeism: the moderating effect of social support. J Appl Psychol 2010; 95:334-48.

4. Holden L, Scuffham PA, Hilton MF, Ware RS, Vecchio N, Whiteford HA. Which health conditions impact on productivity in working Australians? J Occup Environ Med 2011; 53:253-7.

5. Callaghan RC, Sanches M, Gatley JM, Cunningham JK. Effects of the minimum legal drinking age on alcohol-related health service use in hospital settings in Ontario: a regression-discontinuity approach. Am J Public Health 2013; 103:2284-91.

6. World Health Organization. Global status report on alcohol and health 2016. Geneva: World Health Organization; 2016.

7. Lima CT, Farrell M, Prince M. Job strain, hazardous drinking, and alcohol-related disorders. J Stud Alcohol Drugs 2013; 74:212-22.

8. Crum RM, Muntaner C, Eaton WW, Anthony JC. Occupational stress and the risk of alcohol abuse and dependence. Alcohol Clin Exp Res 1995; 19:647-55.

9. Tsutsumi A, Kayaba K, Yoshimura M, Sawada M, Ishikawa S, Sakai K, et al. Association between job characteristics and health behaviors in Japanese rural workers. Int J Behav Med $2003 ; 10: 125-42$. 
10. Frone M. Employee alcohol and illicit drug use: scope, causes, and organizational consequences. In: Quick JC, Tetrick LE, coordinadores. Handbook of occupational health psychology. London: American Psychological Association; 2011. p. 277-96.

11. Bobak M, Pikhart H, Kubinova R, Malyutina S, Pajak A, Sebakova H, et al. The association between psychosocial characteristics at work and problem drinking: a cross-sectional study of men in three Eastern European urban populations. Occup Eviron Med 2005; 62:546-50.

12. Head J, Stansfeld SA, Siegrist J. The psychosocial work environment and alcohol dependence: a prospective study. Occup Environ Med 2004; 61:219-24.

13. Kouvonen A, Kivimäki M, Virtanen M, Heponiemi T, Elovainio M, Pentti J, et al. Effort-reward imbalance at work and the co-ocurrence of lifestyle risk factors: cross-sectional survey in a sample of 36,127 public sector employees. BMC Public Health 2006; 6:24.

14. Ansoleaga E, Montaño R, Vézina M. Psychosocial risk at work and hazardous alcohol consumption among Chile's working adults. Can J Public Health 2013; 104:e502-8.

15. Hemmingsson T, Lundberg I. Work control, work demands, and work social support in relation to alcoholism among young men. Alcohol Clin Exp Res 1998; 22:921-7.

16. Gimeno D, Amick BC, Barrientos-Gutiérrez T, Mangione TW. Work organization and drinking: an epidemiological comparison of two psychosocial work exposure models. Int Arch Occup Environ Health 2009; 82:305-17.

17. Kouvonen A, Kivimäki M, Väänänen A, Heponiemi T, Elovainio M, Ala-Mursula L, et al. Job strain and adverse health behaviors: the Finnish Public Sector Study. J Occup Environ Med 2007; 49:68-74.

18. Cooper ML, Russell M, Frone M. Work stress and alcohol effects: a test of stress-induced drinking. J Health Soc Behav1990; 31:260-76.

19. Romelslö A, Hasin D, Hilton M, Boström G, Diderichsen F, Haglund B, et al. The relationship between stressful working conditions and high alcohol consumption and severe alcohol problems in an urban general population. $\mathrm{Br} \mathrm{J}$ Addict 1992; 87:1173-83.

20. Kouvonen A, Kivimäki M, Elovainio M,Virtanen M, Linna A, Vahtera J. Job strain and leisure-time physical activity in female and male public sector employees. Prev Med 2005; 41:532-9.

21. Demerouti E, Bakker AB, Nachreiner F, Schaufeli WB. The job demands-resources model of burnout. J Appl Psychol 2001; 86:499-512.

22. Bakker AB, Demerouti E, Sanz-Vergel AI Burnout and work engagement: the JD-R approach. Annual Review of Organizational Psychology and Organizational Behavior 2014; $1: 389-411$

23. Tajfel H. Grupos humanos y categorías sociales. Barcelona: Herder; 1984.
24. Tajfel H, Turner JC. La teoría de la identidad social de la conducta intergrupal. En: Morales JF, Huici C, coordinadores. Lecturas de psicología social. Madrid: Universidad Nacional de Educación a Distancia; 1989. p. 41-87.

25. Frone M, Russell M, Copper ML. Job stressors, job involvement and employee heath: a test of identity theory. J Occup Organ Psychol 1995; 68:1-11.

26. Biron M, Bamberger PA, Noyman T. Workrelated risk factors and employee substance use: insights from a sample of Israeli bluecollar workers. J Occup Health Psychol. 2011; 16:247-63.

27. Rinker DV, Neighbors C. Do different types of social identity moderate the association between perceived descriptive norms and drinking among college students? Addict Behav 2014; 39:1297-303.

28. Neighbors C, LaBrie JW, Hummer JF, Lewis MA, Lee CM, Desai S, et al. Group identification as a moderator of the relationship between perceived social norms and alcohol consumption. Psychol Addict Behav 2010; 24:522-8.

29. Tarrant M, Hagger MS, Farrow CV. Promoting positive oirientatio towards health throuh social identity. In: Jetten J, Haslam C, Haslam SA, coordinadores. The social cure: identity, health and well-being. New York: Psychology Press; 2012. p. 39-54.

30. Tarrant M, Butler K. Effects of self-categorization on orientation towards health. $\mathrm{Br} \mathrm{J}$ Soc Psychol 2011; 50:121-39.

31. Grossbard JR, Geisner IM, Mastroleo NR, Kilmer JR, Turrisi R, Larimer ME. Athletic identity, descriptive norms, and drinking among athletes transitioning to college. Addict Behav 2009; 34:352-9.

32. Reed MB, Lange JE, Ketchie JM, Clapp JD. The relationship between social identity, normative information, and college student drinking. Soc Influ 2007; 2:269-94.

33. Baka L. Does job burnout mediate negative effects of job demands on mental and psysical health in a group of teachers? Testing the energetic process of Job Demands-Resources model. Int J Occup Med Environ Health 2015; 28:335-46.

34. Hakanen JJ, Schaufeli WB, Ahola K. The Job Demands-Resources model: a three-year cross-lagged study of burnout, depression, commitment, and work engagement. Work Stress 2008; 22:224-41.

35. Liu C, Nauta NM, Li C, Fan J. Comparisons of organizational constraints and their relations to strains in China and the United States. J Occup Health Psychol 2010; 15:452-67.

36. Copeland MP, Reynolds KJ, Burton JB. Social identity, status characteristics and social networks: Predictors of advice seeking in a manufacturin facility. Asian J Soc Psychol 2008; 11:75-87.

37. Reynolds KJ. Advancing group research: the (non) necessity of behavioral data? Small Group Research 2011; 42:359-73. 
38. Reynolds KJ, Subasic E, Tindall K. The problem of behaviour change: from social norms to an ingroup focus. Soc Personal Psychol Compass 2015; 9:45-56.

39. McAuliffe BJ, Jetten J. Hornsey MJ, Hogg MA. Individualist and collectivist norms: when it's ok to go your own way. Eur J Soc Psychol 2003; 3357-70.

40. Postmes T, Spears R, Lea M. The formation of group norms in computer-mediated communication. Hum Commun Res 2000; 26:341-71.

41. Turner J. Social influence. London: Open University Press; 1991.

42. Cialdini RB, Reno RR, Kallgren CA. A focus theory of normative conduct: recycling the concept of norms to reduce littering in public places. J Pers Soc Psychol 1990; 58:1015-26.

43. Rivis A, Sheeran P. Descriptive norms as an additional predictor in the theory of planned behaviour: a meta-analysis. Curr Psychol 2003; 22:218-33

44. Cialdini RB. The focus theory of normative conduct. In: Van Lange P, Kruglanski A, Higgins $\mathrm{T}$, coordinadores. Handbook of theories of social psychology. v. 2. London: Sage; 2012. p. 295-312.

45. Lapinski MK, Rimal RN. An explication of social norms. Commun Theory 2005; 15:127-47.

46. Mael F, Ashforth BE. Alumni and their alma mater: a partial test of the reformulated model of organizational identification. J Organ Behav 1992; 13:103-23.

47. Dick R, Wagner U, Stellmacher J, Christ O. The utility of a broader conceptualization of organizational identification: which aspects really matter? J Occup Organ Psychol 2004; 77:171-91.

48. Brewer MB. Optimal distinctiveness, social identity, and the self. In: Leary MR, Price J, coordinadores. Handbook of self and identity. New York: Guilford Press; 2003.p. 480-91.

49. van Knippenberg D, van Dich R, Tavares S. Social identity and social exchange: identification, support, and withdrawal from the job. J Appl Soc Psychol 2007; 37:457-77.

50. Riketta M. Organizational identification: a meta-analysis. J Vocat Behav 2005; 66:358-84.

51. Riketta M, van Dick R. Foci of attachment in organizations: a meta-analytic comparison of the strength and correlates of workgroup versus organizational identification and commitment. J Vocat Behav 2005; 67:490-510.

52. García-Guiu C, Molero F, Moya M, Moriano JA. Authentic leadership, group cohesion and group identification in security and emergency teams. Psicothema 2015; 27:59-64.

53. Yang L-Q, Johnson RE, Zhang X, Spector PE, $\mathrm{Xu} \mathrm{S}$. Relations of interpersonal unfairness with counterproductive work behavior: the moderating role of employee self-identity. J Bus Psychol 2013; 28:189-202.

54. Hurtado D, Kawachi I, Sudarsky J. Social capital and self-rated health in Colombia: the good, the bad and the ugly. Soc Sci Med 2011; 72:584-90.
55. Haslam N, Bastian B, Bissett M. Essentialist beliefs about personality and their implications. Pers Soc Psychol Bull 2004; 30:1661-73.

56. León OG, Montero I. Diseño de investigaciones. Introducción a la lógica de la investigación en psicología y educación. 2a Ed. Madrid: McGraw-Hill Interamericana; 1997.

57. Alvarado ME, Garmendia ML, Acuña G, Santis $\mathrm{R}$, Arteaga O. Validez y confiabilidad de la versión chilena del Alcohol Use Disorders Identification Test (AUDIT). Rev Méd Chile 2009; 137:1463-8.

58. Siegrist J, Starke D, Chandola T, Godin I, Marmot M, Niedhammer I, et al. The measurement of effort-reward imbalance at work: European comparisons. Soc Sci Med 2004; 58:1483-99.

59. Güilgüiruca M, Zúñiga C, Quiñones M. Condiciones del trabajo y consumo de alcohol: una relación moderada por la identificación con el grupo de trabajo y la norma de consumo [Tesis de Doctorado]. Santiago: Universidad de Chile; 2017.

60. Alvarado R, Pérez-Franco J, Saavedra N, Fuentealba C, Alarcón A, Marchetti N, et al. Validación de un cuestionario para evaluar riesgos psicosociales en el ambiente laboral en Chile. Rev Méd Chile 2012; 140:1154-63.

61. Postmes T, Haslam SA, Jans L. A single-item measure of social identification: reliability, validity, and utility. Br J Soc Psychol 2013; 52:597-617.

62. Rimal RN, Real K. How behaviors are influenced by perceived norms. Communic Res 2005; 32:389-414.

63. Graham K, Massak A, Demers A, Rehm J. Does the association between alcohol consumption and depression depend on how they are measured? Alcohol Clin Exp Res 2007; 31:78-88.

64. Sieri S, Agudo A, Kesse E, Klipstein-Grobusch K, San-José B, Welch A, et al. Patterns of alcohol consumption in 10 European countries participating in the European Prospective Investigation into Cancer and Nutrition (EPIC) project. Public Health Nutr 2002; 5:1287-96.

65. Byrne BM. Structural equation modeling with Mplus:basic concepts, applications, and programming. New York: Taylor \& Francis/Routledge; 2012.

66. Hayes AF, Preacher KJ. Statistical mediation analysis with a multicategorical indepedient variable. Br J Math Stat Psychol 2014; 67:45170.

67. Dawson JF, Richter AW. Probing three-way interactions in moderated multiple regression: development and application of a slope difference test. J Appl Psychol 2006; 91:917-26.

68. Teunissen HA, Spijkerman R, Cohen GL, Prinstein MJ, Engels RCME, Scholte RHJ. An experimental study on the effects of peer drinking norms on adolescents' drinker prototypes. Addict Behav 2014; 39:85-93.

69. Johnson MB. Experimental test of social norms theory in a real-world drinking environment. J Stud Alcohol Drugs 2012; 73:851-9.

70. Reis AM, Quintal C, Lourenço O. Problemas com bebida no passado coíbem consumo atual? Cad Saúde Pública 2019; 35:e00025618. 


\section{Abstract}

The current study aimed to determine whether workgroup identification and alcohol consumption social norms of workgroup moderates the relationship between job demands and alcohol consumption in workers. Regression analysis using the moderated moderation macro in a sample of workers from a telecommunications company in Chile $(N=1,143)$ supports the hypothesis of moderated moderation: high workgroup identification with with unfavorable social norms of alcohol consumption by the group buffered the relationship between job demands and alcohol consumption of workers $(B=-0.080 ; t=-2.01 ; p=0.04 ; 95 \% \mathrm{CI}$ : -0.1600; -0.0024), and the high workgroup identification with favorable social norms of alcohol consumption by the group increases the relationship between job demands and alcohol consumption of workers $(B=0.170 ; t=2.36 ; p=0.01$; 95\%CI: 0.030; 0.322). The results suggest that strong identification with workgroup and their social norms for alcohol consumption are job resources that can jointly affect or protect workers exposed to heavy work demands. Attention should thus be focused on the potential positive versus harmful effects of these work resources.

Occupational Stress; Alcohol Drinking; Social Identification; Social Norms

\section{Resumo}

O objetivo do presente estudo foi determinar se a identificação com grupos de trabalho, com normas desfavoráveis e favoráveis de consumo de álcool do grupo de trabalho, medeia a relação entre as exigências do trabalho e o consumo de álcool dos trabalhadores. Uma análise de regressão, usando a macro de mediação moderada numa amostra de trabalhadores de uma empresa de telecomunicações do Chile $(N=1.143)$, sustenta as hipóteses de mediação moderada: a alta identificação com grupos de trabalho com normas desfavoráveis de consumo de álcool desse grupo mitiga a relação entre as exigências do trabalho e o consumo de álcool nos trabalhadores $(B=-0,080 ; t=-2,01 ; p=0,04$; IC95\%:-0,1600; -0,0024) e a alta identificação com grupos de trabalho com normas favoráveis de consumo de álcool desse grupo incrementa a relação entre as exigências do trabalho e o consumo de álcool nos trabalhadores $(B=0,170 ; t=2,36$; $p=0,01$; IC95\%: 0,030; 0,322). Estes resultados sugerem que a alta identificação com grupos de trabalho e suas normas sociais de consumo de álcool são recursos laborais que, de maneira conjunta, podem afetar ou proteger trabalhadores expostos a altas exigências laborais. Portanto, recomendam-se considerar os potenciais efeitos positivos, como também os efeitos perniciosos, desses recursos laborais.

Estresse Ocupacional; Consumo de Bebidas Alcoólicas; Identificação Social; Normas Sociais
Recibido el 05/Jul/2019

Versión final presentada el 04/Ene/2020 Aprobado el 14/Feb/2020 